\title{
Properties of the subsemigroups of the bicyclic monoid
}

\author{
L. Descalço \\ Departamento de Matemática, Universidade de Aveiro \\ 3810-193 Aveiro, Portugal \\ N. Ruškuc \\ Mathematical Institute, University of St Andrews \\ St Andrews KY16 9SS, Scotland, U.K.
}

\begin{abstract}
In this paper we study some properties of the subsemigroups of the bicyclic monoid $\mathbf{B}$, by using a recent description of its subsemigroups. We start by giving necessary and sufficient conditions for a subsemigroup to be finitely generated. Then we show that all finitely generated subsemigroups are automatic and finitely presented. Finally we prove that a subsemigroup of $\mathbf{B}$ is residually finite if and only if it does not contain a copy of $\mathbf{B}$.
\end{abstract}

AMS Mathematics Subject Classification: 20M05.

Key words: Bicyclic monoid, subsemigroup, generators, defining relations, automatic structures.

\section{Introduction and Previous Relevant Results}

The bicyclic monoid $\mathbf{B}$, defined by the presentation $\langle b, c \mid b c=1\rangle$, is one of the most fundamental semigroups, with many remarkable properties and generalizations; see $[1,2,5,8,9,12,13,15,16]$.

In this paper we use the description of the subsemigroups of the bicyclic monoid, obtained in [4], to establish some of their properties. We start by giving necessary and sufficient conditions for a subsemigroup to be finitely generated 


\begin{tabular}{c|c|c|c|c}
\multicolumn{1}{c}{0} & \multicolumn{1}{c}{1} & \multicolumn{1}{c}{2} \\
\cline { 2 - 5 } 0 & 1 & $b$ & $b^{2}$ & \\
\cline { 2 - 5 } 1 & $c$ & $c b$ & $c b^{2}$ & \\
\cline { 2 - 5 } & $c^{2}$ & $c^{2} b$ & $c^{2} b^{2}$ & \\
\hline & & & &
\end{tabular}

Figure 1: The bicyclic monoid

(Section 2). Then we show that all finitely generated subsemigroups are automatic and finitely presented (Sections 3,4). Finally we prove that a subsemigroup of $\mathbf{B}$ is residually finite if and only if it does not contain a copy of $\mathbf{B}$ (Section 5).

We begin by introducing the notation that will be used throughout the paper and state some useful known results about the subsemigroups of B. From the defining presentation for $\mathbf{B}$ it is easy to see that every element of $\mathbf{B}$ can be expressed uniquely as $c^{i} b^{j}$ with $i, j \geq 0$. In what follows we shall identify $\mathbf{B}$ with the set $\left\{c^{i} b^{j}: i, j \geq 0\right\}$, and the multiplication then becomes:

$$
c^{i} b^{j} c^{k} b^{l}=\left\{\begin{array}{l}
c^{i-j+k} b^{l} \text { if } j \leq k \\
c^{i} b^{j-k+l} \text { if } j>k .
\end{array}\right.
$$

It is often convenient to view $\mathbf{B}$ as an infinite square grid, as shown in Figure 1. The following three functions $\Phi, \Psi, \lambda: \mathbf{B} \rightarrow \mathbb{N}_{0}, \Phi\left(c^{i} b^{j}\right)=i, \Psi\left(c^{i} b^{j}\right)=j$ and $\lambda\left(c^{i} b^{j}\right)=|j-i|$ will be used extensively throughtout the paper. ( $\Phi$ and $\Psi$ are the first and the second projections respectively, while $\lambda$ is the modulus of the natural epimorphism from $\mathbf{B}$ onto the additive group $\mathbb{Z}$.)

Let us now introduce some basic subsets of $\mathbf{B}$ :

$$
\begin{aligned}
& D=\left\{c^{i} b^{i}: i \geq 0\right\}-\text { the diagonal, } U=\left\{c^{i} b^{j}: j>i \geq 0\right\}-\text { the upper half, } \\
& \left.E_{p}=\left\{c^{i} b^{j}: 0 \leq j<p, i \geq 0\right\}-\text { the left strip (determined by } p \geq 0\right) .
\end{aligned}
$$

Next, consider the function $\widehat{\widehat{A}}: \mathbf{B} \rightarrow \mathbf{B}$ by $c^{i} b^{j} \mapsto \widehat{c^{i} b^{j}}=c^{j} b^{i}$. Geometrically $\widehat{\wedge}$ is the reflection with respect to the main diagonal. So, for example, $\widehat{U}$ is the lower half. Algebraically this function is an anti-isomorphism $(\widehat{x y}=\widehat{y} \widehat{x})$, as is easy to check.

By using the above basic sets and functions we now define some further subsets of $\mathbf{B}$. For $0 \leq q \leq p \leq m$ we define the triangle

$$
T_{q, p}=\left\{c^{i} b^{j}: q \leq i \leq j<p\right\},
$$


and the strips

$$
\begin{aligned}
& S_{q, p}=\left\{c^{i} b^{j}: q \leq i<p, j \geq p\right\}, S_{q, p}^{\prime}=\left\{c^{i} b^{j}: q \leq i<p, j \geq i\right\}, \\
& S_{q, p, m}=\left\{c^{i} b^{j}: q \leq i<p, j \geq m\right\} .
\end{aligned}
$$

Note that for $q=p$ the above sets are empty. For $i, m \geq 0$ and $d>0$ we define the lines

$$
\Lambda_{i}=\left\{c^{i} b^{j}: j \geq 0\right\}, \Lambda_{i, m, d}=\left\{c^{i} b^{j}: d \mid j-i, j \geq m\right\}
$$

and, in general, for $I \subseteq\{0, \ldots, m-1\}$,

$$
\Lambda_{I, m, d}=\bigcup_{i \in I} \Lambda_{i, m, d}=\left\{c^{i} b^{j}: i \in I, d \mid j-i, j \geq m\right\}
$$

For $p \geq 0, d>0, r \in[d]=\{0, \ldots, d-1\}$ and $P \subseteq[d]$ we define the squares

$$
\begin{aligned}
& \Sigma_{p}=\left\{c^{i} b^{j}: i, j \geq p\right\}, \quad \Sigma_{p, d, r}=\left\{c^{p+r+u d} b^{p+r+v d}: u, v \geq 0\right\}, \\
& \Sigma_{p, d, P}=\bigcup_{r \in P} \Sigma_{p, d, r}=\left\{c^{p+r+u d} b^{p+r+v d}: r \in P ; u, v \geq 0\right\} .
\end{aligned}
$$

Figures illustrating some of these sets can be found in [4].

Finally, for $X \subseteq \mathbf{B}$, we define $\iota(X)=\min (\Phi(X \cap U)$ ) (if $X \cap U \neq \emptyset$ ) and $\kappa(X)=\min (\Psi(X \cap \widehat{U}))($ if $X \cap \widehat{U} \neq \emptyset)$.

We can now state the main result from [4], which gives a description of all subsemigroups of the bicyclic monoid:

Proposition 1.1 ([4, Theorem 3.1]) Let $S$ be a subsemigroup of the bicyclic monoid. Then one of the following conditions holds:

1. $S$ is a subset of the diagonal $D$.

2. $S$ is a union of a subset of a triangle, a subset of the diagonal above the triangle, a square below the triangle and some lines belonging to a strip determined by square and the triangle, or the reflection of this union with respect to the diagonal. Formally there exist $q, p \in \mathbb{N}_{0}$ with $q \leq p, d \in \mathbb{N}$, $I \subseteq\{q, \ldots, p-1\}$ with $q \in I, P \subseteq\{0, \ldots, d-1\}$ with $0 \in P, F_{D} \subseteq D \cap E_{q}$, $F \subseteq T_{q, p}$ such that $S$ is of one of the following forms:

(i) $S=F_{D} \cup F \cup \Lambda_{I, p, d} \cup \Sigma_{p, d, P}$; or

(ii) $S=F_{D} \cup \widehat{F} \cup \widehat{\Lambda_{I, p, d}} \cup \Sigma_{p, d, P}$.

3. There exist $d \in \mathbb{N}, I \subseteq \mathbb{N}_{0}, F_{D} \subseteq D \cap E_{\min (I)}$ and sets $S_{i} \subseteq \Lambda_{i, i, d}(i \in I)$ such that $S$ is of one of the following forms: 


$$
\begin{aligned}
& \text { (i) } S=F_{D} \cup \bigcup_{i \in I} S_{i} \text {; or } \\
& \text { (ii) } S=F_{D} \cup \bigcup_{i \in I} \widehat{S}_{i} \text {; }
\end{aligned}
$$

where each $S_{i}$ has the form

$$
S_{i}=F_{i} \cup \Lambda_{i, m_{i}, d}
$$

for some $m_{i} \in \mathbb{N}_{0}$ and some finite set $F_{i}$, and

$$
I=I_{0} \cup\left\{r+u d: r \in R, u \in \mathbb{N}_{0}, r+u d \geq N\right\}
$$

for some (possibly empty) $R \subseteq\{0, \ldots, d-1\}$, some $N \in \mathbb{N}_{0}$ and some finite set $I_{0} \subseteq\{0, \ldots, N-1\}$.

We call diagonal subsemigroups those satisfying 1, two-sided subsemigroups those satisfying 2, upper subsemigroups those satisfying 3 (i) and lower subsemigroups those satisfying 3 (ii). Figures 2 and 3 illustrate the several kinds of subsemigroups, by giving some examples.

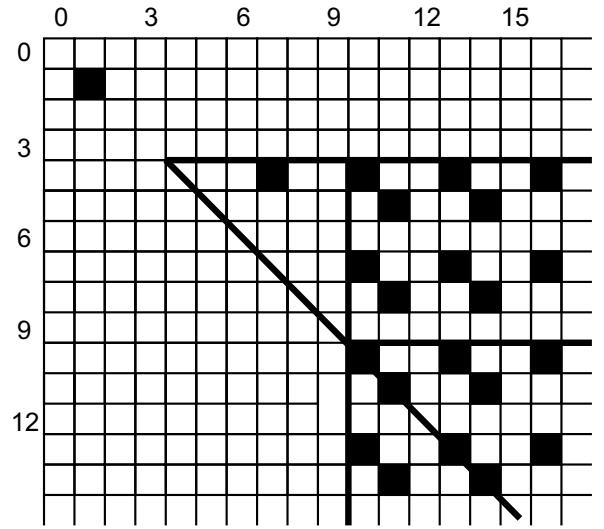

(a) $d=3, F_{D}=\{c b\}, F=\left\{c^{4} b^{7}\right\}$, $I=\{4,5,7,8\}, p=10, P=\{0,1\}$

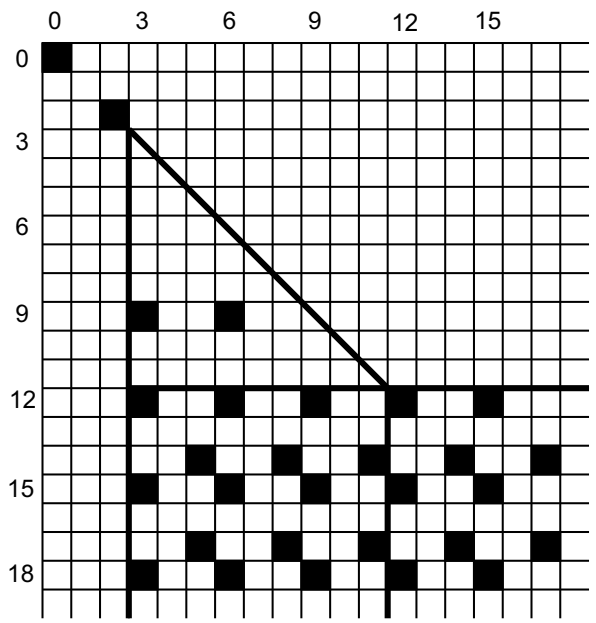

(b) $d=3, F_{D}=\left\{1, c^{2} b^{2}\right\}, p=12$, $F=\left\{c^{3} b^{9}, c^{6} b^{9}\right\}, P=\{0,2\}$, $I=\{3,5,6,8,9,11\}$

Figure 2: Two-sided subsemigroups

Observation 1.2 In the case where $I$ is finite $(R=\emptyset)$, an upper subsemigroup can be written as a union of two finite sets and finitely many lines all starting 


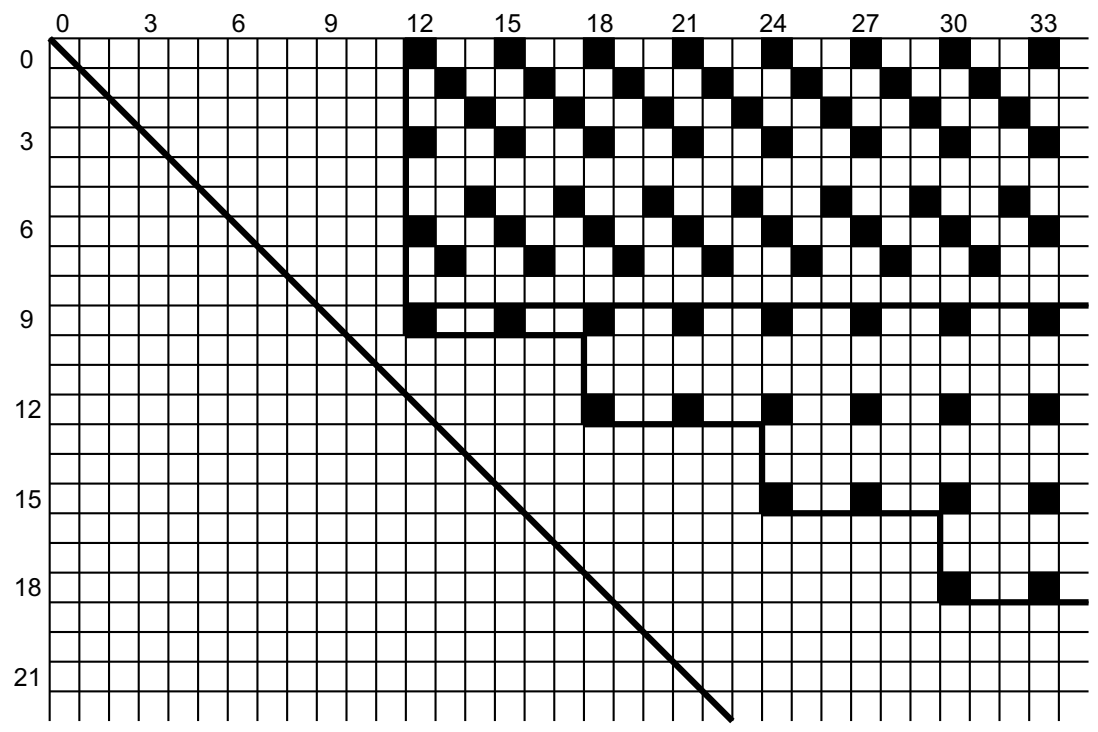

(a) $d=3, F_{D}=\emptyset, I_{0}=\{0,1,2,3,5,6,7\}, I=I_{0} \cup\{9+3 u: u \geq 0\}$, $F_{i}=0(i \in I), m_{i}=12\left(i \in I_{0}\right), m_{i}=2 i-6\left(i \in I \backslash I_{0}\right)$

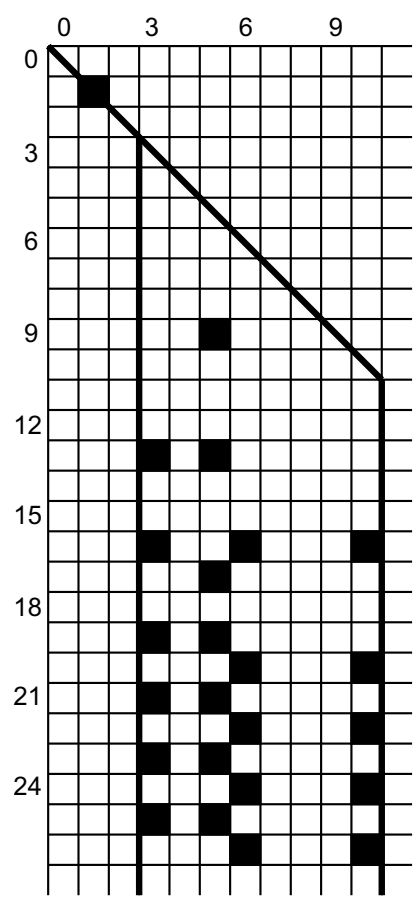

(b) $d=2, I=\{3,5,6,10\}, F_{D}=\{c b\}, m_{3}=19, F_{3}=\left\{c^{3} b^{13}, c^{3} b^{16}\right\}, m_{5}=17$, $F_{5}=\left\{c^{5} b^{9}, c^{5} b^{13}\right\}, m_{6}=20, F_{6}=\left\{c^{6} b^{16}\right\}, m_{10}=20, F_{10}=\left\{c^{10} b^{16}\right\}$

Figure 3: Upper and lower subsemigroups 
from the same column. Formally there exist $q, p, m \in \mathbb{N}_{0}$ with $q<p \leq m$, finite sets $F_{D} \subseteq D \cap E_{q}, F \subseteq S_{q, p}^{\prime} \backslash S_{q, p, m}$ and a set $I \subseteq\{q, \ldots, p-1\}$ such that

$$
S=F_{D} \cup F \cup \Lambda_{I, m, d}
$$

A similar observation applies to a lower subsemigroup.

The following result, proved in [4], will also be needed:

Proposition 1.3 ([4, Lemma 4.3]) For any $q, p \in \mathbb{N}_{0}$ with $q \leq p$ the sets $S_{q, p} \cup \Sigma_{p}$ and $S_{q, p}^{\prime}(q<p)$ are subsemigroups of the bicyclic monoid.

\section{Finite generation}

If $A$ is a finite set, we denote by $A^{+}$the free semigroup generated by $A$ consisting of non empty words over $A$ under the concatenation, and by $A^{*}$ the free monoid generated by $A$ consisting of $A^{+}$together with the empty word $\epsilon$. Let $S$ be a semigroup and $\psi: A \rightarrow S$ a mapping. We say that $A$ is a finite generating set for $S$ with respect to $\psi$ if the unique extension of $\psi$ to a semigroup homomorphism $\psi: A^{+} \rightarrow S$ is surjective. For $u, v \in A^{+}$we write $u \equiv v$ to mean that $u$ and $v$ are equal as words and $u=v$ to mean that $u$ and $v$ represent the same element in the semigroup i.e. that $u \psi=v \psi$.

In this section we will establish necessary and sufficient conditions for a subsemigroup of the bicyclic monoid to be finitely generated proving the following:

Theorem 2.1 Let $S$ be a subsemigroup of the bicyclic monoid. Then $S$ is finitely generated if and only if one of the following conditions holds:

(i) $S$ is a finite diagonal subsemigroup;

(ii) $S$ is a two-sided subsemigroup;

(iii) $S$ is an upper subsemigroup and the set $\left\{i \in \mathbb{N}_{0}: E_{i} \cap S \neq \emptyset\right\}$ is finite;

(iv) $S$ is a lower subsemigroup and the set $\left\{i \in \mathbb{N}_{0}: \widehat{E_{i}} \cap S \neq \emptyset\right\}$ is finite.

Proof. (i) Since $c^{i} b^{i} c^{j} b^{j}=c^{k} b^{k}$, with $k=\max (i, j)$, a subsemigroup of the bicyclic monoid contained in the diagonal only admits itself as a generating set, and so it is finitely generated if and only if it is finite. 
(ii) To prove that every two-sided subsemigroup $S$ of $\mathbf{B}$ is finitely generated it is sufficient to note that $S$ can be expressed as a finite disjoint union of copies of $\mathbf{B}$ and subsemigroups of $\mathbb{N}_{0}$; see [4, Theorem 7.6]. Since each of these is finitely generated, it follows that $S$ is finitely generated as well.

For future use, we also give a direct proof of our claim, and in doing so establish a natural finite generating set for $S$. Let $\iota(S)=q$ and $\kappa(S)=p$ and let $d=\operatorname{gcd}(\lambda(S))$. We can assume, without loss of generality, that $q \leq p$. By Theorem 1.1 we have

$$
S=F_{D} \cup F \cup \Sigma_{p, d, P} \cup \Lambda_{I, p, d}
$$

where $F$ and $F_{D}$ are finite sets and $I \subseteq\{q, \ldots, p-1\}$ for some $q, p \in \mathbb{N}_{0}$. For every $i \in I$ let $i+u_{i} d=\min \{i+u d: i+u d \geq p\}$. We will prove that the finite set

$$
Y=\left\{c^{i} b^{i+u_{i} d}: i \in I\right\} \cup\left\{c^{p} b^{p+d}, c^{p+d} b^{p}\right\} \cup\left\{c^{p+r} b^{p+r}: r \in P\right\}
$$

generates the subsemigroup $\Sigma_{p, d, P} \cup \Lambda_{I, p, d}$. Indeed, for $c^{i} b^{i+u d} \in \Lambda_{I, p, d}$ we have $c^{i} b^{i+u d}=c^{i} b^{i+u_{i} d}\left(c^{p} b^{p+d}\right)^{u-u_{i}}$ while for $c^{p+r+u d} b^{p+r+v d} \in \Sigma_{p, d, P}$ we have

$$
c^{p+r+u d} b^{p+r+v d}=\left(c^{p+d} b^{p}\right)^{u}\left(c^{p+r} b^{p+r}\right)\left(c^{p} b^{p+d}\right)^{v} .
$$

Therefore the whole of $S$ can be generated by the finite set $F_{D} \cup F \cup Y$.

(iii) We will prove that an upper semigroup $S$ is finitely generated if and only if the set

$$
K=\left\{i \in \mathbb{N}_{0}: E_{i} \cap S \neq \emptyset\right\}
$$

is finite. We first assume that $K$ is infinite and prove that $S$ is not finitely generated. Suppose that there exists a finite set $X$ such that $S=\langle X\rangle$. Since $X \subseteq S \subseteq U \cup D$ and $X$ is finite, this implies $X \subseteq S_{0, p}^{\prime}$ for some $p \in \mathbb{N}_{0}$. Hence $S=\langle X\rangle \subseteq S_{0, p}^{\prime}$ because, by Proposition 1.3, $S_{0, p}^{\prime}$ is a subsemigroup, and therefore $K \subseteq\{0, \ldots, p\}$ is finite, which contradicts our assumption. We conclude that $S$ is not finitely generated.

If we now assume that $K$ is finite then to prove that $S$ is finitely generated it suffices to observe that $S$ is a finite union of subsemigroups of the infinite monogenic semigroup $\mathbb{N}$ (one in each line).

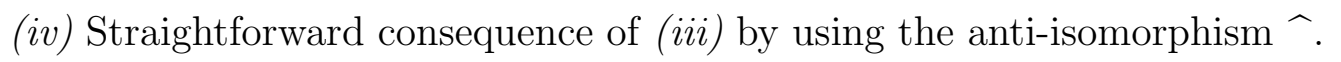




\section{Automaticity}

Given a finite set $A$, and a subset $L$ of $A^{+}$we say that $L$ is regular if there is a finite state automaton accepting it, and we say that $L$ is rational if it can be obtained from finite subsets of $A^{*}$ by finitely many applications of $\cup$ (union), . (concatenation) and $*$ (Klenne's star operation). It is well known that notions of 'regular' and 'rational' coincide and we use them as synonyms. To be able to deal with automata that accept pairs of words and to define automatic semigroups we need to define a new alphabet $A(2, \$)=((A \cup\{\$\}) \times(A \cup\{\$\})) \backslash\{(\$, \$)\}$ where $\$$ is a symbol not in $A$ (called the padding symbol) and the function $\delta_{A}: A^{*} \times A^{*} \rightarrow$ $A(2, \$)^{*}$ defined by

$$
\left(a_{1} \ldots a_{m}, b_{1} \ldots b_{n}\right) \delta_{A}= \begin{cases}\epsilon & \text { if } 0=m=n \\ \left(a_{1}, b_{1}\right) \ldots\left(a_{m}, b_{m}\right) & \text { if } 0<m=n \\ \left(a_{1}, b_{1}\right) \ldots\left(a_{m}, b_{m}\right)\left(\$, b_{m+1}\right) \ldots\left(\$, b_{n}\right) & \text { if } 0 \leq m<n \\ \left(a_{1}, b_{1}\right) \ldots\left(a_{n}, b_{n}\right)\left(a_{n+1}, \$\right) \ldots\left(a_{m}, \$\right) & \text { if } m>n \geq 0 .\end{cases}
$$

Let $S$ be a semigroup and let $A$ be a finite generating set for $S$ with respect to $\psi: A^{+} \rightarrow S$. The pair $(A, L)$ is an automatic structure for $S$ (with respect to $\psi$ ) if

- $L$ is a regular subset of $A^{+}$and $L \psi=S$,

- $L_{=}=\{(\alpha, \beta): \alpha, \beta \in L, \alpha=\beta\} \delta_{A}$ is regular in $A(2, \$)^{+}$, and

- $L_{a}=\{(\alpha, \beta): \alpha, \beta \in L, \alpha a=\beta\} \delta_{A}$ is regular in $A(2, \$)^{+}$for each $a \in A$,

where, as before, $\alpha=\beta$ means that $\alpha$ and $\beta$ represent the same element in $S$ (i.e. $\alpha \psi=\beta \psi$ ). We say that a semigroup is automatic if it has an automatic structure. For a more detailed introduction see [3].

If $(A, L)$ is an automatic structure for a semigroup $S$ then there is an automatic structure $(A, K)$ such that each element of $S$ has a unique representative in $K$ (see [3, Proposition 5.4]); we say that $(A, K)$ is an automatic structure with uniqueness and that $K$ is a set of unique normal forms for $S$.

In this section we will consider automaticity of the subsemigroups of the bicyclic monoid and our main result is the following:

Theorem 3.1 All finitely generated subsemigroups of the bicyclic monoid are automatic. 
A finitely generated subsemigroup of $\mathbf{B}$ is a finite union of subsemigroups of $\mathbb{N}$ and copies of $\mathbf{B}$. However, it is not known whether a finite union of automatic semigroups is necessarily automatic. Hence we need to devise a direct proof of Theorem 3.1. A major ingredient is the following general result from [6]:

Proposition 3.2 ([6, Theorem 1.1]) Let $S$ be a semigroup and let $T$ be a subsemigroup of $S$ such that the set $S \backslash T$ is finite. Then $S$ is automatic if and only if $T$ is automatic.

This result will be combined with the following:

Lemma 3.3 For any numbers $p, m \in \mathbb{N}_{0}$ with $p \leq m, d \in \mathbb{N}$ and sets $I \subseteq$ $\{0, \ldots, p-1\}, P \subseteq\{0, \ldots, d-1\}$ such that $0 \in P$, each of the following subsets of the bicyclic monoid is automatic whenever it is a subsemigroup:

$$
\begin{array}{ll}
\text { (i) } \Lambda_{I, m, d} ; & \text { (ii) } \widehat{\Lambda_{I, m, d}} ; \\
\text { (iii) } \quad \Sigma_{p, d, P} \cup \Lambda_{I, p, d} ; & \text { (iv) } \Sigma_{p, d, P} \cup \widehat{\Lambda_{I, p, d}}
\end{array}
$$

ProOF. We observe that although the the semigroups (ii) and (iv) are obtained

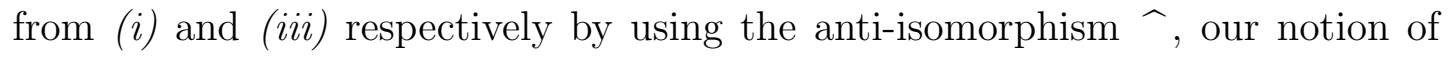
automatic structure involves multiplication on the right and so we cannot just apply to obtain the latter automatic structures and we need to prove each of the four cases separately. (In [7], four alternative definitions of automatic semigroup are studied, that correspond to the use of right or left multiplication and to the use of the padding symbol on the right or on the left. These definitions are equivalent when applied to groups but, as shown in [7], they are completely independent for semigroups.)

(i) Let $i+u_{i} d=\min \{i+u d: i+u d \geq m\}$ for $i \in I$. Fixing $i_{0} \in I$ and $u=u_{i_{0}}$ we define the alphabet

$$
\Lambda=\bigcup_{i \in I}\{\lambda(i, 0), \ldots, \lambda(i, u-1)\}
$$

and the homomorphism

$$
f: \Lambda^{*} \rightarrow \Lambda_{I, m, d} ; \lambda(i, j) \mapsto c^{i} b^{i+\left(u_{i}+j\right) d}
$$

Defining

$$
L=\bigcup_{i \in I}\left(\bigcup_{j=0}^{u-1}\left\{\lambda(i, j) \lambda\left(i_{0}, 0\right)^{n}: n \geq 0\right\}\right)
$$


it is clear that $L$ is a regular language and we will show that it is a set of unique normal forms for $S=\Lambda_{I, m, d}$. Given $s \in S$ we can write $s=c^{i} b^{i+\left(u_{i}+k\right) d}$ for some $i \in I$ and $k \geq 0$. Dividing $k$ by $u$ we obtain $k=n u+j$ with $n \geq 0$ and $0 \leq j<u$ and hence the unique word in $L$ representing $s$ is the word $\lambda(i, j) \lambda\left(i_{0}, 0\right)^{n}$. To prove that the pair $(\Lambda, L)$ is an automatic structure for $S$ we only have to show that the languages

$$
L_{\lambda(k, l)}=\left\{\left(w_{1}, w_{2}\right) \delta: w_{1}, w_{2} \in L, w_{1} \lambda(k, l)=w_{2}\right\}
$$

are regular for every $\lambda(k, l) \in \Lambda$. We can write

$$
\lambda(i, j) \lambda\left(i_{0}, 0\right)^{n} \lambda(k, l)=c^{i} b^{i+\left(u_{i}+j\right) d+n u d} c^{k} b^{k+\left(u_{k}+l\right) d}=c^{i} b^{i+\left(u_{i}+j+u_{k}+l\right) d+n u d}
$$

and dividing $j+u_{k}+l$ by $u$ we obtain $j+u_{k}+l=q u+r$ with $q \geq 0$ and $0 \leq r<u$ and so we have

$$
\lambda(i, j) \lambda\left(i_{0}, 0\right)^{n} \lambda(k, l)=c^{i} b^{i+\left(u_{i}+r\right) d+(n+q) u d}=\lambda(i, r) \lambda\left(i_{0}, 0\right)^{n+q}
$$

where $w=s$ with $w \in \Lambda^{*}, s \in S$ means that $w$ represents the element $s$ (i.e. $w f=s)$. Therefore we have

$$
L_{\lambda(k, l)}=\bigcup_{i \in I}\left(\bigcup_{j=0}^{u-1} Y_{k, l, i, j}\right)
$$

where

$$
\begin{aligned}
Y_{k, l, i, j}=\{ & \left(\lambda(i, j) \lambda\left(i_{0}, 0\right)^{n}, \lambda(i, r) \lambda\left(i_{0}, 0\right)^{n+q}\right) \delta: \\
& \left.u_{k}+j+l=q u+r, 0 \leq r<u, n \geq 0\right\} .
\end{aligned}
$$

Each set $Y_{k, l, i, j}$ is regular because the numbers $q$ and $r$ are uniquely determined by the fixed numbers $k, l, i$ and $j$, and we have

$$
Y_{k, l, i, j}=\{(\lambda(i, j), \lambda(i, r))\} \cdot\left\{\left(\lambda\left(i_{0}, 0\right), \lambda\left(i_{0}, 0\right)\right)\right\}^{*} \cdot\left\{\left(\epsilon, \lambda\left(i_{0}, 0\right)^{q}\right) \delta\right\} .
$$

Hence $L_{\lambda(k, l)}$ is regular.

(ii) We define $u_{i}(i \in I), i_{0}, u$ and the alphabet $\Lambda$ as in the proof of (i) but now our homomorphism is

$$
f: \Lambda^{*} \rightarrow S ; \lambda(i, j) \mapsto c^{i+\left(u_{i}+j\right) d} b^{i}
$$

and our regular language is

$$
L=\bigcup_{i \in I}\left(\bigcup_{j=0}^{u-1}\left\{\lambda\left(i_{0}, 0\right)^{n} \lambda(i, j): n \geq 0\right\}\right)
$$


where $S=\widehat{\Lambda_{I, m, d}}$. Again, $L$ is a set of unique normal forms for $S$, since we have $\lambda\left(i_{0}, 0\right)^{n} \lambda(i, j)=c^{i+\left(u_{i}+j\right) d+n u d} b^{i}$, and we will prove that the languages

$$
L_{\lambda(k, l)}=\left\{\left(w_{1}, w_{2}\right) \delta: w_{1}, w_{2} \in L, w_{1} \lambda(k, l)=w_{2}\right\}
$$

are regular for every $\lambda(k, l) \in \Lambda$. We can write

$$
\lambda\left(i_{0}, 0\right)^{n} \lambda(i, j) \lambda(k, l)=c^{i+\left(u_{i}+j\right) d+n u d} b^{i} c^{k+\left(u_{k}+l\right) d} b^{k}=c^{k+\left(u_{k}+j+u_{i}+l\right) d+n u d} b^{k}
$$

and dividing $j+u_{i}+l$ by $u$ we obtain $j+u_{i}+l=q u+r$ with $q \geq 0$ and $0 \leq r<u$ and so we have

$$
\lambda\left(i_{0}, 0\right)^{n} \lambda(i, j) \lambda(k, l)=c^{k+\left(u_{k}+r\right) d+(q+n) u d} b^{k}=\lambda\left(i_{0}, 0\right)^{q+n} \lambda(k, r) .
$$

Therefore we have

$$
\begin{aligned}
& L_{\lambda(k, l)}=\bigcup_{i \in I}\left(\bigcup _ { j = 0 } ^ { u - 1 } \left\{\left(\lambda\left(i_{0}, 0\right)^{n} \lambda(i, j), \lambda\left(i_{0}, 0\right)^{n+q} \lambda(k, r)\right) \delta:\right.\right. \\
& \left.\left.u_{i}+j+l=q u+r, 0 \leq r<u, n \geq 0\right\}\right)
\end{aligned}
$$

which is a finite union of regular languages and so is regular.

(iii) Let $Z=\Lambda \cup\{x, y\} \cup \Gamma$, where $\Lambda=\left\{\lambda_{i}: i \in I\right\}$ and $\Gamma=\left\{\gamma_{r}: r \in P\right\}$, be an alphabet and define

$$
L=\bigcup_{i \in I}\left(\left\{\lambda_{i} x^{u}: u \geq 0\right\}\right) \cup \bigcup_{r \in P}\left(\left\{y^{v} \gamma_{r} x^{u}: u, v \geq 0\right\}\right),
$$

which is a regular subset of $Z^{+}$. We are going to prove that $(Z, L)$ is an automatic structure (with uniqueness) for the semigroup $S=\Sigma_{p, d, P} \cup \Lambda_{I, p, d}$ with respect to

$$
f: Z^{+} \rightarrow S ; \lambda_{i} \mapsto c^{i} b^{i+u_{i} d}, \gamma_{r} \mapsto c^{p+r} b^{p+r}, x \mapsto c^{p} b^{p+d}, y \mapsto c^{p+d} b^{p}
$$

where $i+u_{i} d=\min \{i+u d: i+u d \geq p\}$ for $i \in I$.

To show that each element in $S$ has a unique representative in $L$ it suffices to observe that

$$
\lambda_{i} x^{u}=c^{i} b^{i+\left(u_{i}+u\right) d}(i \in I ; u \geq 0), y^{v} \gamma_{r} x^{u}=c^{p+r+v d} b^{p+r+u d}(r \in P ; u, v \geq 0) .
$$

Therefore we only have to show that that languages $L_{z}=\left\{\left(w_{1}, w_{2}\right) \delta: w_{1}, w_{2} \in\right.$ $\left.L, w_{1} z=w_{2}\right\}$ are regular for every $z \in Z$. We will first consider the case where $z=\lambda_{t} \in \Lambda$. Since $\Psi\left(\left(\lambda_{i} x^{u}\right) f\right), \Psi\left(\left(y^{v} \gamma_{r} x^{u}\right) f\right) \geq p>t=\Phi\left(\lambda_{t} f\right)$ we have

$$
L_{\lambda_{t}}=\bigcup_{i \in I}\left\{\left(\lambda_{i} x^{u}, \lambda_{i} x^{u+u_{t}}\right) \delta: u \geq 0\right\} \cup \bigcup_{r \in P}\left\{\left(y^{u} \gamma_{r} x^{u}, y^{v} \gamma_{r} x^{u+u_{t}}\right) \delta: u, v \geq 0\right\}
$$


which is a regular language. We will now consider $z=\gamma_{t} \in \Gamma$. Since for $u>0$ we have $\Psi\left(\left(\lambda_{i} x^{u}\right) f\right), \Psi\left(\left(y^{v} \gamma_{r} x^{u}\right) f\right) \geq p+d>\Phi\left(\gamma_{t} f\right)$ we have

$$
\begin{gathered}
L_{\gamma_{t}}=\bigcup_{i \in I}\left(\left\{\left(\lambda_{i} x^{u}, \lambda_{i} x^{u}\right) \delta: u>0\right\} \cup\left\{\left(\lambda_{i}, w\right) \delta: w \in L, \lambda_{i} \gamma_{t}=w\right\}\right) \cup \\
\bigcup_{r \in P}\left(\left\{\left(y^{v} \gamma_{r} x^{u}, y^{v} \gamma_{r} x^{u}\right) \delta: v \geq 0, u>0\right\} \cup L_{\left(\gamma_{t}, r\right)}\right)
\end{gathered}
$$

where

$$
L_{\left(\gamma_{t}, r\right)}=\left\{\begin{array}{l}
\left\{\left(y^{u} \gamma_{r}, y^{u} \gamma_{r}\right) \delta: u \geq 0\right\} \text { if } r \geq t \\
\left\{\left(y^{u} \gamma_{r}, y^{u} \gamma_{t}\right) \delta: u \geq 0\right\} \text { otherwise. }
\end{array}\right.
$$

We note that, for each $i \in I$, the set $\left\{\left(\lambda_{i}, w\right) \delta: w \in L, \lambda_{i} \gamma_{t}=w\right\}$ has only one element because $L$ is a set of unique normal forms for $S$, and so the language $L_{\gamma_{t}}$ is a finite union of regular languages and therefore it is regular. The language $L_{x}$ is clearly regular since we have $L_{x}=\{(w, w x) \delta: w \in L\}$. Finally, we have

$$
\begin{aligned}
L_{y}= & \bigcup_{i \in I}\left(\left\{\left(\lambda_{i} x^{u}, \lambda_{i} x^{u-1}\right) \delta: u>0\right\} \cup\left\{\left(\lambda_{i}, w\right) \delta: w \in L, \lambda_{i} y=w\right\}\right) \cup \\
& \bigcup_{r \in P}\left(\left\{\left(y^{v} \gamma_{r} x^{u}, y^{v} \gamma_{r} x^{u-1}\right) \delta: v \geq 0, u>0\right\} \cup\left\{\left(y^{v} \gamma_{r}, y^{v+1} \gamma_{0}\right) \delta: v \geq 0\right\}\right)
\end{aligned}
$$

because, for $v \geq 0$, we have

$$
\left(y^{v} \gamma_{r}\right) y=\left(c^{p+r+v d} b^{p+r}\right)\left(c^{p+d} b^{p}\right)=c^{p+(v+1) d} b^{p}=y^{v+1} \gamma_{0}
$$

Again, for each $i \in I$, the set $\left\{\left(\lambda_{i}, w\right) \delta: w \in L, \lambda_{i} y=w\right\}$ is regular because it has only one element and so $L_{y}$ is also a finite union of regular languages and hence is regular. We conclude that $S$ is automatic.

(iv) We define the alphabet $Z$ as in the proof of (iii) and our regular language over $Z^{+}$is now

$$
L=\bigcup_{i \in I}\left(\left\{y^{v} \lambda_{i}: v \geq 0\right\}\right) \cup \bigcup_{r \in P}\left(\left\{y^{v} \gamma_{r} x^{u}: u, v \geq 0\right\}\right) .
$$

We are going to prove that $(Z, L)$ is an automatic structure (with uniqueness) for the semigroup $S=\Sigma_{p, d, P} \cup \widehat{\Lambda_{I, p, d}}$ with respect to

$$
f: Z^{+} \rightarrow S ; \lambda_{i} \mapsto c^{i+u_{i} d} b^{i}, \gamma_{r} \mapsto c^{p+r} b^{p+r}, x \mapsto c^{p} b^{p+d}, y \mapsto c^{p+d} b^{p}
$$

again with $i+u_{i} d=\min \{i+u d: i+u d \geq p\}$ for $i \in I$.

It is again clear that $L$ is a set of unique normal forms for $S$ and we will show that the languages $L_{z}=\left\{\left(w_{1}, w_{2}\right) \delta: w_{1}, w_{2} \in L, w_{1} z=w_{2}\right\}$ are regular for every $z \in Z$. We start by showing that, for any $\lambda_{t} \in \Lambda$, we have

$$
\begin{aligned}
L_{\lambda_{t}}= & \bigcup_{i \in I}\left\{\left(y^{v} \lambda_{i}, y^{v+u_{i}} \lambda_{t}\right) \delta: v \geq 0\right\} \cup \\
& \bigcup_{r \in P}\left(\left\{\left(y^{v} \gamma_{r} x^{u}, y^{v} \gamma_{r} x^{u-u_{t}}\right) \delta: v \geq 0, u \geq u_{t}\right\} \cup L_{\left(\lambda_{t}, r\right)} \cup\right. \\
& \left.\bigcup_{u=1}^{u_{t}-1}\left\{\left(y^{v} \gamma_{r} x^{u}, y^{v+u_{t}-u-u_{k}} \lambda_{k}\right) \delta: v \geq 0, k=p+r+\left(u-u_{t}\right) d\right\}\right)
\end{aligned}
$$


where

$$
L_{\left(\lambda_{t}, r\right)}=\left\{\begin{array}{l}
\left\{\left(y^{v} \gamma_{r}, y^{v} \lambda_{t}\right) \delta: v \geq 0\right\} \text { if } p+r \leq t+u_{t} d \\
\left\{\left(y^{v} \gamma_{r}, y^{v+u_{t}-u_{k}} \lambda_{k}\right) \delta: k=p+r-u_{t} d\right\} \text { otherwise }
\end{array}\right.
$$

We have

$$
y^{v} \lambda_{i} \lambda_{t}=c^{i+u_{i} d+v d} b^{i} c^{t+u_{t} d} b^{t}=c^{t+u_{t} d+\left(v+u_{i}\right) d} b^{t}=y^{v+u_{i}} \lambda_{t} .
$$

If $u \geq u_{t}$ then

$$
y^{v} \gamma_{r} x^{u} \lambda_{t}=c^{p+r+v d} b^{p+r+u d} c^{t+u_{t} d} b^{t}=c^{p+r+v d} b^{p+r+\left(u-u_{t}\right) d}=y^{v} \gamma_{r} x^{u-u_{t}} .
$$

For $u \in\left\{1, \ldots, u_{t}-1\right\}$ we define $k=p+r+\left(u-u_{t}\right) d$ and we have

$$
\begin{aligned}
w & =y^{v} \gamma_{r} x^{u} \lambda_{t}=c^{p+r+v d} b^{p+r+u d} c^{t+u_{t} d} b^{t}=c^{p+r+v d} b^{p+r+\left(u-u_{t}\right) d} \\
& =c^{k+\left(v+u_{t}-u\right) d} b^{k}=c^{k+u_{k} d+\left(v+u_{t}-u-u_{k}\right) d} b^{k}
\end{aligned}
$$

Since $S$ is a semigroup and $k<p$ we have $w \in \widehat{\Lambda_{I, p, d}}$ and therefore, observing the definition of $u_{k}$, it must be $v+u_{t}-u-u_{k} \geq 0$ and we can write $w=$ $y^{v+u_{t}-u-u_{k}} \lambda_{k}$. We will now consider the multiplication of a word of the form $y^{v} \gamma_{r}$ by $\lambda_{t}$ and so we define $w=y^{v} \gamma_{r} \lambda_{t}=c^{p+r+v d} b^{p+r} c^{t+u_{t} d} b^{t}$. If $p+r \leq t+u_{t} d$ then $w=c^{t+u_{t} d+v d} b^{t}=y^{v} \lambda_{t}$. If $p+r>t+u_{t} d$ we have $w=c^{p+r+v d} b^{p+r-u_{t} d}$. We observe that $u_{t}>0$ because $t<p$ and $t+u_{t} d \geq p$ and therefore $w \in \widehat{\Lambda_{I, p, d}}$. Hence, defining $k=p+r-u_{t} d$ we can write

$$
w=c^{k+\left(v+u_{t}\right) d} b^{k}=c^{k+u_{k} d+\left(v+u_{t}-u_{k}\right) d} b^{k}
$$

and, from the definition of $u_{k}$, it follows that $v+u_{t}-u_{k} \geq 0$ and so we have $w=y^{v+u_{t}-u_{k}} \lambda_{k}$. We conclude that $L_{\lambda_{t}}$ can be defined as a finite union of regular languages and so it is a regular language.

It is easy to see that

$$
\begin{aligned}
L_{\gamma_{t}}= & \bigcup_{i \in I}\left\{\left(y^{v} \lambda_{i}, y^{v+u_{i}} \gamma_{t}\right) \delta: v \geq 0\right\} \cup L_{\left(\gamma_{t}, r\right)} \\
& \bigcup_{r \in P}\left\{\left(y^{v} \gamma_{r} x^{u}, y^{v} \gamma_{r} x^{u}\right) \delta: u>0, v \geq 0\right\}
\end{aligned}
$$

where

$$
L_{\left(\gamma_{t}, r\right)}=\left\{\begin{array}{l}
\left\{\left(y^{v} \gamma_{r}, y^{v} \gamma_{r}\right) \delta: v \leq 0\right\} \text { if } r \geq t \\
\left\{\left(y^{v} \gamma_{r}, y^{v} \gamma_{t}\right) \delta: v \geq 0\right\} \text { otherwise }
\end{array}\right.
$$

and so it is a regular language. The language $L_{x}$ is regular because we have

$$
L_{x}=\bigcup_{i \in I}\left\{\left(y^{v} \lambda_{i}, y^{u_{i}+v} \gamma_{0} x\right) \delta: v \geq 0\right\} \cup \bigcup_{r \in P}\left\{\left(y^{v} \gamma_{r} x^{u}, y^{v} \gamma_{r} x^{u+1}\right) \delta: u, v \geq 0\right\}
$$


and since

$$
\begin{aligned}
L_{y}= & \bigcup_{i \in I}\left\{\left(y^{v} \lambda_{i}, y^{v+u_{i}+1} \gamma_{0}\right) \delta: v \geq 0\right\} \cup \\
& \bigcup_{r \in P}\left(\left\{\left(y^{v} \gamma_{r} x^{u}, y^{v} \gamma_{r} x^{u-1}\right) \delta: v \geq 0, u>0\right\} \cup\left\{\left(y^{v} \gamma_{r}, y^{v+1} \gamma_{0}\right) \delta: v \geq 0\right\}\right)
\end{aligned}
$$

$L_{y}$ is a regular language as well. We conclude that $(Z, L)$ is an automatic structure for $S$.

Proof of TheOREM 3.1 We know from the previous section that any finitely generated subsemigroup is either a finite subset of the diagonal, and so it is automatic, or it has one of the forms:

$$
\begin{aligned}
& F_{D} \cup F \cup \Lambda_{I, p, d} \cup \Sigma_{p, d, P}, F_{D} \cup F \cup \widehat{\Lambda_{I, p, d}} \cup \Sigma_{p, d, P}, \\
& F_{D} \cup F \cup \Lambda_{I, p, d}, \quad F_{D} \cup F \cup \widehat{\Lambda_{I, p, d}}
\end{aligned}
$$

where $I \subseteq\{q, q+1, \ldots, p-1\}$ for some numbers $q, p \in \mathbb{N}_{0}$ and the sets $F$ and $F_{D}$ are finite. In each case we can remove the finite set $F_{D} \cup F$ from our subsemigroup and we still have a subsemigroup, because we are in fact intersecting it with the set $S_{q, p} \cup \Sigma_{p}$, which by Proposition 1.1 is itself a subsemigroup. Hence every finitely generated subsemigroup $S$ of $\mathbf{B}$ has a subsemigroup $U$ such that $S \backslash U$ is finite and that, by the previous lemma, is automatic. It follows from Proposition 1.3 that $S$ is automatic as well.

\section{$4 \quad$ Finite presentability}

Let $A$ be an alphabet and let $R \subseteq A^{+} \times A^{+}$be a relation on $A^{+}$. We say that the semigroup $S$ is defined by the presentation $\langle A \mid R\rangle$ if $S$ is generated by $A$ with respect to a mapping $\psi: A \rightarrow S$, and the kernel of the extension of $\psi$ to a homomorphism $A^{+} \rightarrow S$ is the smallest congruence $\rho$ containing $R$. In this case, of course, we have $S \cong A^{+} / \rho$. Given a presentation $\langle A \mid R\rangle$, for two words $w, z \in A^{+}$we write $w \rightarrow^{*} z$, and say that $w=z$ is a consequence of $R$ (or that the word $w$ can be reduced to $z$ by applying relations from $R$ ), to mean that either $w \equiv v$ or that there is a sequence a words $w \equiv w_{1}, w_{2}, \ldots, w_{n} \equiv v$ where for each $i=1, \ldots, n-1$ we can write $w_{i} \equiv \alpha_{i} u_{i} \beta_{i}, w_{i+1} \equiv \alpha_{i} v_{i} \beta_{i}$ for some $\alpha_{i}, \beta_{i} \in A^{*}$ and $\left(u_{i}, v_{i}\right) \in R$ or $\left(v_{i}, u_{i}\right) \in R$. It is then well known that the relation $w=z$ holds in $S$ (i.e. $w \psi=z \psi$ ) if and only if it is a consequence of $R$. Moreover, given a semigroup $S$ generated by a set $A$ and a set $R \subseteq A^{+} \times A^{+}$, the pair $\langle A \mid R\rangle$ 
is a presentation for $S$ if and only if $S$ satisfies all relations from $R$ and any other relation that holds in $S$ is a consequence of $R$. We will use the following straightforward consequence of this fact:

Proposition 4.1 Let $S$ be a semigroup generated by a set $A$, let $R \subseteq A^{+} \times A^{+}$ and let $L \subseteq A^{+}$be a set of unique normal forms for $S$. If the following conditions hold:

(i) S satisfies all the relations from $R$; and

(ii) any word $w \in A^{+}$can be reduced to the corresponding unique normal form in $L$ by using relations from $R$;

then $\langle A \mid R\rangle$ is a presentation for $S$.

We say that a semigroup $S$ is finitely presented if there is a presentation $\langle A \mid R\rangle$ for $S$ where both $A$ and $R$ are finite sets. For further details about semigroup presentations we refer the reader to [10].

In the previous section Proposition 3.2 allowed us to remove finite subsets from the semigroups when considering automaticity. We have a similar result for finite presentability, proved in [14]:

Proposition 4.2 ([14, Theorem 1.3]) Let $S$ be a semigroup and $T$ be a subsemigroup of $S$ such that $S \backslash T$ is finite. Then $S$ is finitely presented if and only if $T$ is finitely presented.

Our main result of this section is the following:

Theorem 4.3 All finitely generated subsemigroups of the bicyclic monoid are finitely presented.

The main work on the proof of Theorem 4.3 is contained in the following:

Lemma 4.4 For any numbers $p, m \in \mathbb{N}_{0}$ with $p \leq m, d \in \mathbb{N}$ and sets $I \subseteq$ $\{0, \ldots, p-1\}, P \subseteq\{0, \ldots, d-1\}$ such that $0 \in P$, each of the following subsets of $\mathbf{B}$ is finitely presented whenever it is a subsemigroup:
(i) $\Lambda_{I, m, d}$;
(ii) $\Lambda_{I, p, d} \cup \Sigma_{p, d, P}$ 
Proof. (i) We consider the automatic structure $(\Lambda, L)$ obtained in the proof of Lemma 3.3 (i), which gives us a finite generating set and a set of unique normal forms for $\Lambda_{I, m, d}$. We are going to prove that $\langle\Lambda \mid R\rangle$ is a finite presentation for $T$, where $R$ consists of the following relations:

$$
\begin{aligned}
& \lambda(i, j) \lambda(k, l)=\lambda(i, r) \lambda\left(i_{0}, 0\right)^{q} \text { where } j+u_{k}+l=q u+r, 0 \leq r<u \\
& (i, k \in I, j, l \in\{0, \ldots, u-1\}) .
\end{aligned}
$$

That the relations hold follows from equation (1), in the proof of Lemma 3.3. We are going to show that any word $w \in \Lambda^{+}$can be reduced to a word in $L$ by applying relations from $R$, using induction on the length $|w|$ of the word $w$. If $|w|=1$ then $w \in L$ by definition of $L$. If $|w|=2$ then $w=\lambda(i, j) \lambda(k, l)$ and therefore

$$
w \rightarrow^{*} \lambda(i, r) \lambda\left(i_{0}, 0\right)^{q} \in L, j+u_{k}+l=q u+r(0 \leq r<u)
$$

using one relation from $R$. Let $n \geq 2$ and suppose that any word $w$ such that $|w| \leq n$ can be reduced to a word in $L$ by using relations from $R$. Let $w \in \Lambda^{+}$ with $|w|=n+1$. We have $w=\lambda\left(i_{1}, j_{1}\right) \ldots \lambda\left(i_{n}, j_{n}\right) \lambda\left(i_{n+1}, j_{n+1}\right)$. We can reduce $\lambda\left(i_{n}, j_{n}\right) \lambda\left(i_{n+1}, j_{n+1}\right)$ obtaining

$$
w \rightarrow^{*} \lambda\left(i_{1}, j_{1}\right) \ldots \lambda\left(i_{n-1}, j_{n-1}\right) \lambda\left(i_{n}, r\right) \lambda\left(i_{0}, 0\right)^{q}
$$

where

$$
j_{n}+u_{i_{n+1}}+j_{n+1}=q u+r(0 \leq r<u) .
$$

Letting $w^{\prime}=\lambda\left(i_{1}, j_{1}\right) \ldots \lambda\left(i_{n-1}, j_{n-1}\right) \lambda\left(i_{n}, r\right)$ we have $\left|w^{\prime}\right|=n$ and, using the induction hypothesis, we have $w^{\prime} \rightarrow^{*} \lambda(i, j) \lambda\left(i_{0}, 0\right)^{m} \in L$ for some $i \in I, j \in$ $\{0, \ldots, u-1\}, m \in \mathbb{N}_{0}$, implying $w \rightarrow^{*} \lambda(i, j) \lambda\left(i_{0}, 0\right)^{m+q} \in L$.

(ii) We will use the automatic structure $(Z, L)$ obtained in the proof of Lemma 3.3 (iii) to prove that $T=\Sigma_{p, d, P} \cup \Lambda_{I, p, d}$ is finitely presented. We will show that $<Z \mid R>$ is a finite presentation for $T$, defining $R$ to be the following set of relations:

$$
\begin{aligned}
x & =\gamma_{0} x \\
y & =y \gamma_{0} \\
\lambda_{i} \lambda_{j} & =\lambda_{i} x^{u_{j}} \quad(i, j \in I) \\
x \lambda_{i} & =x^{1+u_{i}} \quad(i \in I) \\
y \lambda_{i} & =y x^{u_{i}} \quad(i \in I)
\end{aligned}
$$




$$
\begin{aligned}
\gamma_{r} \lambda_{i} & =\gamma_{r} x^{u_{i}} \quad(r \in P, i \in I) \\
x y & =\gamma_{0} \\
\lambda_{i} y & =\lambda_{j} \quad\left(i \in I, u_{i}>1, j=p+d-u_{i} d\right) \\
\lambda_{i} y & =\gamma_{0} \quad\left(i \in I, u_{i}=1\right) \\
\gamma_{r} y & =y \quad(r \in P) \\
x \gamma_{r} & =x \quad(r \in P) \\
\lambda_{i} \gamma_{r} & =\lambda_{i} \quad\left(i \in I, r \in P, i+u_{i} d \geq p+r\right) \\
\lambda_{i} \gamma_{r} & =\lambda_{j} \quad\left(i \in I, r \in P, i+u_{i} d<p+r, j=p+r-u_{i} d\right) \\
\gamma_{r} \gamma_{t} & =\gamma_{r} \quad(r \geq t) \\
\gamma_{r} \gamma_{t} & =\gamma_{t} \quad(r<t)
\end{aligned}
$$

To see that any of these relations holds we just have to prove that both sides of it correspond to the same word in $\left\{c^{i} b^{j}: i, j \geq 0\right\}$. We will only prove that relations (9), (10), (13) and (14) hold since for the others this is straightforward.

To prove that relations (9) and (10) hold we observe that, by definition of $u_{i}$, we have $\lambda_{i} y=c^{i} b^{i+u_{i} d} c^{p+d} b^{p}=c^{p+d-u_{i} d} b^{p}$. If $u_{i}=1$ then $\lambda_{i} y=c^{p} b^{p}=\gamma_{0}$ and relation (10) holds. If $u_{i}>1$ then $p+d-u_{i} d<p$ and so, defining $j=p+d-u_{i} d$, we have $\lambda_{i} y=c^{j} b^{j+\left(u_{i}-1\right) d} \in \Lambda_{I, p, d}$. But we have $j+\left(u_{i}-1\right) d=p$ which implies, by definition of $u_{j}$, that $u_{i}-1=u_{j}$ which means that $\lambda_{i} y=\lambda_{j}$ and relation (9) holds as well.

To prove that relations (13) and (14) hold we start by writing

$$
\lambda_{i} \gamma_{r}=c^{i} b^{i+u_{i} d} c^{p+r} b^{p+r}
$$

If $i+u_{i} d \geq p+r$ then $\lambda_{i} \gamma_{r}=c^{i} b^{i+u_{i} d}=\lambda_{i}$ and relation (13) holds. Otherwise we have $\lambda_{i} \gamma_{r}=c^{p+r-u_{i} d} b^{p+r} \in \Lambda_{I, p, d}$ because $u_{i}>0$. Defining $j=p+r-u_{i} d$ we have $\lambda_{i} \gamma_{r}=c^{j} b^{j+u_{i} d}$ and, since $j+u_{i} d=p+r<p+d$ and using the definition of $u_{j}$, we must have $u_{i}=u_{j}$, which implies $\lambda_{i} \gamma_{r}=\lambda_{j}$ and relation (14) holds as well.

We are now going to prove that any word in $w \in Z^{+}$can be reduced to a word in $L$, using our relations, by induction on the length of $w$. If $|w|=1$ then either $w \in L$ or it can be reduced to a word in $L$ by using one of the relations (2) or (3). We now consider words of length 2 . The word $\lambda_{i} \lambda_{t}$ reduces to $\lambda_{i} x^{u_{t}} \in L$ using relation (4); $\lambda_{i} x \in L ; \lambda_{i} y$ either reduces to $\gamma_{0} \in L$ using relation (10) or to $\lambda_{j} \in L$ for some $j$ using relation (9); $\lambda_{i} \gamma_{r}$ reduces to $\lambda_{j} \in L$ for some $j$ using relations (13) or (14); $x x$ reduces to $\gamma_{0} x^{2} \in L$ using (2); $x y$ reduces to $\gamma_{0} \in L$ 
using relation (8); $x \lambda_{i}$ reduces to $\gamma_{0} x^{1+u_{i}} \in L$ using relations (5) and (2); $x \gamma_{t}$ reduces to $\gamma_{0} x \in L$ using relations (12) and (2); yx reduces to $y \gamma_{0} x \in L$ using (2); yy reduces to $y^{2} \gamma_{0} \in L$ using (3); y $\lambda_{i}$ reduces to $y \gamma_{0} x^{u_{i}} \in L$ using (6) and (3); $y \gamma_{t} \in L ; \gamma_{i} x \in L ; \gamma_{i} y$ reduces to $y \gamma_{0} \in L$ using (11) and (3); $\gamma_{i} \lambda_{t}$ reduces to $\gamma_{i} x^{u_{t}} \in L$ using (7); finally $\gamma_{i} \gamma_{r}$ reduces to $\gamma_{j} \in L$ for some $j$ using (15) or (16).

In the following induction step we use that fact that if a word $w$ belongs to $L$ then $w x^{n}$ belongs to $L$ as well for any $n \in \mathbb{N}_{0}$, which follows immediately from the definition of $L$. Let $n \geq 2$ and suppose that all words $w \in Z^{+}$with $|w| \leq n$ can be reduced to a word in $L$. Let $w \in Z^{+}$be a word of length $n+1$. Then we have $w=w_{1} g_{1} g_{2}$ with $w_{1} \in Z^{+}$and $g_{1}, g_{2} \in Z$. We will consider all possible pairs of generators $g_{1}, g_{2} \in Z$ and prove that in every case $w$ reduces to a word in $L$ using the relations.

Case 1: $g_{1} g_{2} \in\left\{\lambda_{i} y, \lambda_{i} \gamma_{t}, x y, x \gamma_{t}, \gamma_{t} y, \gamma_{t} \gamma_{i}\right\}$. In these cases we can apply one of the relations to reduce $g_{1} g_{2}$ to a generator $g$. We can then apply the induction hypothesis to reduce $w_{1} g$ to a word in $L$.

Case 2: $g_{1} g_{2} \equiv g_{1} x$. In these cases we can reduce $w_{1} g_{1}$ to a word $w_{2} \in L$ using the induction hypothesis and so we can reduce $w$ to $w_{2} x \in L$.

Case 3: $g_{1} g_{2} \equiv \lambda_{i} \lambda_{t}$. Using relation (4) we have $w \rightarrow^{*} w_{1} \lambda_{i} x^{u_{t}}$ and, since $\left|w_{1} \lambda_{i}\right|=n$, using the induction hypothesis we have $w_{1} \lambda_{i} \rightarrow^{*} w_{2} \in L$ and therefore $w \rightarrow^{*} w_{2} x^{u_{t}} \in L$.

Case 4: $g_{1} g_{2} \equiv x \lambda_{t}$. Using relation (5) we have $w \rightarrow^{*} w_{1} x^{1+u_{t}}$. Since $\left|w_{1}\right| \leq n$, using the hypothesis we can write $w_{1} \rightarrow^{*} w_{2} \in L$ and so $w \rightarrow^{*} w_{1} x^{1+u_{t}} \rightarrow^{*}$ $w_{2} x^{1+u_{t}} \in L$.

Case 5: $g_{1} g_{2} \equiv y \lambda_{t}$. Using relation (6) we reduce $y \lambda_{t}$ to $y x^{u_{t}}$. We can then apply the induction hypothesis to $w_{1} y$ to obtain $w_{1} y \rightarrow^{*} w_{2} \in L$ implying $w \rightarrow^{*} w_{2} x^{u_{t}} \in L$.

Case 6: $g_{1} g_{2} \equiv y y$. We start by reducing $w_{1} y$ to a word $w_{2} \in L$ using the induction hypothesis. We can have $w_{2} \equiv \lambda_{i} x^{u}$ or $w_{2} \equiv y^{v} \gamma_{r} x^{u}$. If $w_{2} \equiv \lambda_{i}$ then $w \rightarrow^{*} \lambda_{i} y$ and applying relations (9) or (10) it reduces to a word in $L$. If $w_{2} \equiv \lambda_{i} x$ then $w \rightarrow^{*} \lambda_{i} x y \rightarrow^{*} \lambda_{i} \gamma_{0}$ by applying relation (8). Therefore by applying now relations (13) or (14), $w$ reduces to word in $L$. If $w_{2} \equiv \lambda_{i} x^{u}$ with $u>1$ then

$$
w \rightarrow^{*} \lambda_{i} x^{u-1} x y \rightarrow^{*} \lambda_{i} x^{u-2} x \gamma_{0} \rightarrow^{*} \lambda_{i} x^{u-1} \in L,
$$

by applying relations (8) and (12). If $w_{2} \equiv y^{v} \gamma_{r}$ then

$$
w \rightarrow^{*} y^{v} \gamma_{r} y \rightarrow^{*} y^{v} y \rightarrow^{*} y^{v+1} \gamma_{0} \in L
$$


using relations (11) and (3). If $w_{2} \equiv y^{v} \gamma_{r} x$ then $w \rightarrow^{*} y^{v} \gamma_{r} x y$ and we can apply relation (8) to reduce $x y$ to $\gamma_{0}$. Then we can reduce $\gamma_{r} \gamma_{0}$ to $\gamma_{r}$ by applying relation (15) and so $w \rightarrow^{*} y^{v} \gamma_{r} \in L$. We can have $w_{2} \equiv y^{v} \gamma_{r} x^{u}$ with $u>1$ and then

$$
w \rightarrow^{*} y^{v} \gamma_{r} x^{u-1} x y \rightarrow^{*} y^{v} \gamma_{r} x^{u-2} x \gamma_{0} \rightarrow^{*} y^{v} \gamma_{r} x^{u-1} \in L
$$

by applying relations (8) and (12).

Case \%: $g_{1} g_{2} \equiv y \gamma_{t}$. We start again by reducing $w_{1} y$ to a word $w_{2} \in L$. We can have $w_{2} \equiv \lambda_{i} x^{u}$ or $w_{2} \equiv y^{v} \gamma_{r} x^{u}$. If $w_{2} \equiv \lambda_{i}$ then $w \rightarrow^{*} \lambda_{i} y$ and applying relation (9) or relation (10) we can reduce $w$ to a generator that belongs to $L$. If $w_{2} \equiv \lambda_{i} x^{u}$ with $u>0$ then we can apply relation (12) giving $w \rightarrow^{*} \lambda_{i} x^{u} \gamma_{t} \rightarrow^{*} \lambda_{i} x^{u} \in L$. If $w_{2} \equiv y^{v} \gamma_{r}$ then $w \rightarrow^{*} y^{v} \gamma_{r} \gamma_{t}$ and so applying relations (15) or (16) we have $w \rightarrow^{*} y^{v} g \in L$ with $g \in\left\{\gamma_{r}, \gamma_{t}\right\}$. Finally, if $w_{2} \equiv y^{v} \gamma_{r} x^{u}$ with $u>0$ then we have $w \rightarrow^{*} y^{v} \gamma_{r} x^{u} \gamma_{t} \rightarrow^{*} y^{v} \gamma_{r} x^{u} \in L$ by applying relation (12).

Case 8: $g_{1} g_{2} \equiv \gamma_{t} \lambda_{i}$. Applying relation (7) we get $\gamma_{t} \lambda_{i} \rightarrow^{*} \gamma_{t} x^{u_{i}}$. Since $\left|w_{1} \gamma_{t}\right| \leq n$, using the hypothesis, we have $w_{1} \gamma_{t} \rightarrow^{*} w_{2} \in L$ and so $w \rightarrow^{*} w_{2} x^{u_{i}} \in$ $L$.

Proof of Theorem 4.3 We know from Section 1 that any finitely generated subsemigroup is either a finite subset of the diagonal, and so it is finitely presented, or it has one of the forms:

$$
\begin{aligned}
& F_{D} \cup F \cup \Lambda_{I, p, d} \cup \Sigma_{p, d, P}, \quad F_{D} \cup F \cup \widehat{\Lambda_{I, p, d}} \cup \Sigma_{p, d, P}, \\
& F_{D} \cup F \cup \Lambda_{I, p, d}, \quad F_{D} \cup F \cup \widehat{\Lambda_{I, p, d}}
\end{aligned}
$$

where $I \subseteq\{q, q+1, \ldots, p-1\}$ for some numbers $q, p \in \mathbb{N}_{0}$ and the sets $F$ and $F_{D}$ are finite. Without loss of generality we may consider only subsemigroups of the form

$$
F_{D} \cup F \cup \Lambda_{I, p, d} \cup \Sigma_{p, d, P}, F_{D} \cup F \cup \Lambda_{I, p, d}
$$

(the other two are anti-isomorphic to them). In both cases we can remove the finite set $F_{D} \cup F$ from our subsemigroup and we still have a subsemigroup. Hence, in both cases, our subsemigroup $S$ has a subsemigroup $U$ such that $S \backslash U$ is finite and which, by Lemma 4.4, is finitely presented. It follows from Proposition 4.2 that $S$ is finitely presented as well. 


\section{Residual finiteness}

We say that a semigroup $S$ is residually finite if, for any two elements $s_{1}, s_{2} \in S$, there is a finite semigroup $F$ and a homomorphism $\phi: S \rightarrow F$ that separates $s_{1}$ and $s_{2}$ (such that $s_{1} \phi \neq s_{2} \phi$ ). We have the following:

Theorem 5.1 A subsemigroup of the bicyclic monoid $\mathbf{B}$ is residually finite if and only if it is not two-sided.

ProOF. We first show that a two-sided semigroup is not residually finite. In fact, a two-sided semigroup $S$ contains a subset of the form $X=\left\{c^{p+u d} b^{p+v d} ; u, v \geq 0\right\}$, which is isomorphic to the bicyclic monoid; the mapping $\psi: \mathbf{B} \rightarrow X ; c^{u} b^{v} \mapsto$ $c^{p+u d} b^{p+v d}$ is clearly an isomorphism. Since $\mathbf{B}$ is not residually finite (see [11]) it follows that $S$ is not residually finite either.

We will now show that a subsemigroup $S$ contained in $U$ (an upper semigroup or a subset of the diagonal) is residually finite. Let $\alpha=c^{i} b^{j}$ and $\beta=c^{k} b^{l}$ be two arbitrary elements of $S$. Taking $p \geq \max (j, l)$ the set $S_{p}=S \cap I_{p}$ is an ideal of $S$. Hence the Rees homomorphism $\phi: S \rightarrow\left(S \backslash S_{p}\right) \cup\{0\}$ separates $\alpha$ and $\beta$, and $S \backslash S_{p} \cup\{0\}$ is finite, since $S \backslash S_{p} \subseteq T_{0, p}$. Analogously, any subsemigroup contained in $\widehat{U}$ is residually finite.

This theorem has the following equivalent formulation:

Theorem 5.2 A subsemigroup of the bicyclic monoid $\mathbf{B}$ is residually finite if and only if it does not contain a copy of $\mathbf{B}$.

Acknowledgements. The first author acknowledges the support of the Fundação para a Ciência e a Tecnologia, Portugal (grant number PRAXIS XXI/BD/19730/99). The second author acknowledges partial financial support of INTAS 99/1224.

\section{References}

[1] C. L. Adair, A generalization of the bicyclic semigroup, Semigroup Forum 21 (1980), 13-25.

[2] K. Byleen, J. Meakin and F. Pastijn, The fundamental four-spiral semigroup, J. Algebra 54 (1978), 6-26. 
[3] C. M. Campbell, E. F. Robertson, N. Ruškuc and R. M. Thomas, Automatic semigroups, Theoret. Comput. Sci. 250 (2001), 365-391.

[4] L. Descalço and N. Ruškuc, Subsemigroups of the bicyclic monoid, Internat. J. Algebra Comput. 15 (2005), 37-57.

[5] P. A. Grillet, On the fundamental double four-spiral semigroup, Bull. Belg. Math. Soc. Simon Stevin 3 (1996), 201-208.

[6] M. Hoffmann, N. Ruškuc and R. M. Thomas, Automatic semigroups with subsemigroups of finite Rees index, Internat. J. Algebra Comput. 12 (2002), 463-476.

[7] M. Hoffmann and R. M. Thomas, Notions of automaticity in semigroups, Semigroup Forum 66 (2003), 337-367.

[8] J. W. Hogan, The $\alpha$-bicyclic semigroup as a topological semigroup, Semigroup Forum 28 (1984), 265-271.

[9] J. M. Howie, Fundamentals of Semigroup Theory, Oxford University Press, 1995.

[10] G. Lallement, Semigroups and Combinatorial Applications, John Wiley \& Sons, 1979.

[11] G. Lallement, On monoids presented by a single relation, J. Algebra 32 (1974), 370-388.

[12] M. V. Lawson, Inverse Semigroups, World Scientific, 1998.

[13] S. O. Makanjuola and A. Umar, On a certain subsemigroup of the bicyclic semigroup, Comm. Algebra 25 (1997), 509-519.

[14] N. Ruškuc, On large subsemigroups and finiteness conditions of semigroups, Proc. London Math. Soc. (76) (1998), 383-405.

[15] L. N. Shevrin, The bicyclic semigroup is determined by its subsemigroup lattice, Bull. Belg. Math. Soc. Simon Stevin 67 (1993), 49-53.

[16] L. N. Shevrin and A. J. Ovsyannikov, Semigroups and Their Subsemigroup Lattices, Kluwer Academic Publishers, 1996. 